\title{
Hypopnea definitions, determinants and dilemmas: a focused review
}

\author{
Q. Afifa Shamim-Uzzaman ${ }^{1 *}$, Sukhmani Singh ${ }^{2}$ and Susmita Chowdhuri ${ }^{3}$
}

\begin{abstract}
Obstructive sleep apnea (OSA) is defined by the presence of repetitive obstructive apneas and hypopneas during sleep. While apneas are clearly defined as cessation of flow, controversy has plagued the many definitions of hypopneas, which have used variable criteria for reductions in flow, with or without the presence of electroencephalographic (EEG) arousal, and with varying degrees of oxygen desaturation. While the prevalence of OSA is estimated to vary using the different definitions of hypopneas, the impact of these variable definitions on clinical outcomes is not clear. This focused review examines the controversies and limitations surrounding the different definitions of hypopnea, evaluates the impact of hypopneas and different hypopnea definitions on clinical outcomes, identifies gaps in research surrounding hypopneas, and makes suggestions for future research.
\end{abstract}

Keywords: Obstructive sleep apnea, Hypopnea, Obstructive hypopnea, Central hypopnea

\section{Introduction}

Obstructive sleep apnea (OSA) is a common disorder, composed of apneas and hypopneas occurring at least five times per hour during sleep. Since polysomnographic identification in 1965, the notion of apneas (absence of airflow for $>10 \mathrm{~s}$, Fig. 1) remains undisputed; however, the definition of hypopneas continues to evolve and their clinical impact debated over the years.

Bloch et al. first described 'hypopneas' as reductions in oxygen saturation that occurred in association with reductions in airflow instead of with absence of airflow, i.e., events suggestive of decreased ventilation that did not meet criteria for apneas. (Bloch et al., 1979) In this study "normal" asymptomatic volunteers had $40 \%$ more hypopneas than apneas (105 vs. 60, respectively) with frequent oxygen desaturation of $\geq 4 \%$. (Bloch et al., 1979) Subsequently, in a small study comparing individuals with apneas alone vs. hypopneas alone $(n=50)$, Gould et al. noted no differences in age, weight, clinical symptoms, number of arousals (median $31 / \mathrm{h}$ vs. $20 / \mathrm{h}$ ) or patterns of oxygen desaturation (median 45 vs. $40,4 \%$ desaturation per hour) (Gould et al., 1988) between the two groups, and recommended changing the terminology

\footnotetext{
* Correspondence: afifa@med.umich.edu

${ }^{1}$ VA Ann Arbor Heathcare Center and University of Michigan, 2215 Fuller Rd, Ann Arbor, MI 48105, USA

Full list of author information is available at the end of the article
}

from "sleep apnea syndrome" to "sleep hypopnea syndrome," defined as 15 or more hypopneas per hour of sleep in conjunction with 2 or more major clinical features. Although the term "sleep hypopnea syndrome" did not gain much popularity, the terminology "sleep apneahypopnea syndrome" (SAHS) was used frequently, until the current term "obstructive sleep apnea" gained favor.

\section{Objectives}

In this focused review, our objective was to describe the variability in the definitions of hypopneas, limitations of technology that are used to detect hypopneas, and thereafter, make suggestions for future research to standardize hypopnea definition and detection. Our literature review also attempted to identify the potential clinical relevance of patients with hypopnea-predominant sleep apnea. These are outlined below.

\section{Background}

\section{Defining moments for 'hypopnea'}

Gould's definition of hypopnea was derived by comparing $75,50 \%$ or $25 \%$ reductions in Respitrace thoracoabdominal sum compared to thermocouple flow amplitude with arousal frequency and oxygen desaturations. (Gould et al., 1988) In this study, a 75\% reduction in movement resulted in much fewer hypopneas than the number of desaturations or arousals and was excluded 


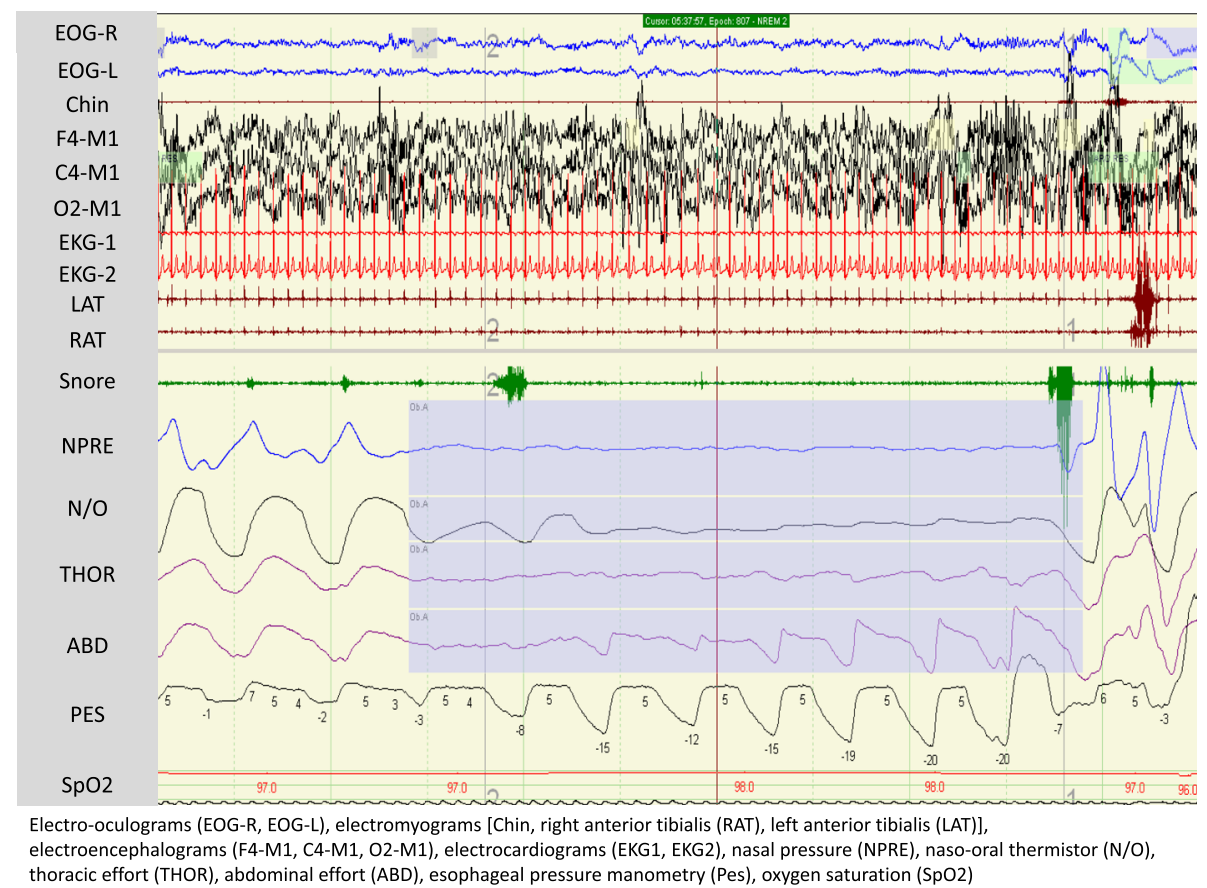

Fig. 1 This figure shows an obstructive apnea. An apnea is a respiratory event lasting $\geq 10 \mathrm{~s}$, characterized by a decrement in airflow of $\geq 90 \%$ from the baseline in the oronasal thermocouple signal. Clear crescendo effort in the abdominal belt suggests obstruction. Elevated and progressively increasing values in the $\Delta$ Pes during the event confirm the obstructive etiology

from consideration. While reductions in thoraco-abdominal movement of $25-50 \%$ were of similar accuracy and more accurate than the frequency of oxygen desaturation alone, the $50 \%$ reduction in effort was significantly closer to the arousal frequency than was the $25 \%$ reduction in thoracoabdominal movement $(p<0.05)$. Hence, these authors defined 'hypopnea' as a " $50 \%$ reduction in thoracoabdominal (Respitrace sum) amplitude for 10 seconds or more when compared to the peak amplitude lasting for 10s or more that occurred within the previous 2 minutes in the presence of continued flow". (Gould et al., 1988)

In 1997, the AASM created a task force to delineate the criteria to identify and treat OSA. Their results, presented as a consensus statement commonly referred to as the "Chicago Criteria," defined hypopnea as a $\geq 50 \%$ decrement in airflow, or a $<50 \%$ reduction in airflow associated with either an oxygen desaturation or arousal. (Loube et al., 1999) Despite this, no uniform definition of 'hypopnea' was used amongst sleep laboratories within the United States for the next decade. (Moser et al., 1994; Redline \& Sanders, 1997) A survey of 44 accredited sleep laboratories (labs) showed as many methods and definitions of hypopneas as number of labs. (Moser et al., 1994) Methods of detection included use of thermocouple, pneumotachograph, respiratory inductance plethysmography, intercostal electromyography, microphone or esophageal balloon. Additionally, the requirements for the degree of airflow reduction and oxygen desaturation also varied widely. Moreover, 33 of the 44 labs used EEG arousal to fulfill the definition of hypopnea, even though there was no consistent definition of arousal at that time. This lack of precision precluded objective comparison of data from individual laboratories and raised doubts to the validity and reproducibility of hypopneas even within the same individual. In fact, Redline et al. (Redline et al., 2000) examined the effect of using 11 different criteria for scoring hypopneas on the prevalence of disease in a large community-based sample and reported that different approaches for measuring apnea-hypopnea index (AHI: number of apneas and hypopneas per hour of sleep) resulted in substantial variability in identifying and classifying sleep-disordered breathing.

\section{Findings}

\section{A. Sources of variability in hypopnea detection}

i) Variability in flow measurements: Hypopnea detection implies determination of small changes in ventilation that accompany sleep disordered breathing; the amplitude of airflow is a measure of these changes. Sources of variability that contribute to poor reliability of these measurements of airflow include:

1) positioning of thermo-elements, as slight displacements could produce major changes in signal amplitude, 
2) alterations in proportion between nasal and oral breathing,

3) nasal cycle causing alterations in nasal airflow (which could change with changes in body position), (Cole \& Haight, 1986)

4) variation in sensitivity and frequency response between different thermo-elements, (Berg et al., 1997)

5) displacement of the Respitrace ${ }^{\circ}$ girdles that could alter signal amplitude.

ii) Type of device: Variability can also arise from the type of devices used during the recording. One study demonstrated that despite relatively high correlation coefficients between the methods of detecting hypopneas, agreement between the devices detecting changes in ventilation (using thermistor, nasal pressure and/or Respitrace ${ }^{\circ}$ ) were low, with poor agreement with minute ventilation measured by head-out body plethysmography in awake subjects. (Berg et al., 1997) The best agreement was noted with plethysmographic minute ventilations and the amplitudes of the summed Respitrace signals, and from the nasal-pressure signals. In fact, nasal-pressure measurements provided the greatest sensitivity and negative predictive values. Combination of nasal pressure and Respitrace ${ }^{\bullet}$ provided more consistent results $-86 \%$ sensitivity and $83 \%$ specificity - and better agreement between both methods (Cohen's $\mathrm{K}=0.65$ ).

iii) Observer reproducibility: Finally, Whyte et al. showed reproducibility in scoring of hypopneas by different observers. (Whyte et al., 1992) When two polysomnographers were asked to independently score both apneas and hypopneas on all-night polysomnograms of patients with OSA using the same methodology, there was close agreement between the polysomnographers for the number of hypopneas ( $r=0.98$; mean difference $11 \%$ ) and for the number of apneas $(r=0.99$; mean difference $8 \%)$. The agreement was similar for the durations of both hypopneas ( $r=0.99$; mean difference 13\%) and apneas $(r=0.99$; mean difference $11 \%)$. There was also close agreement between the total number of respiratory events scored with and without reference to the flow signal $(r=0.99$; mean difference $1.4 \%)$ with a maximum under-recognition of 18 events per night in a subject with 237 apneas per night. (Whyte et al., 1992) Hence, it was possible for different observers to score hypopneas reliably.

iv) Variability in baseline: The lack of clear determination of "baseline" or normative values for each patient lends itself to inherent variability. If the baseline (SpO2, flow, EEG, muscle tone, etc) is not clear, variations from the baseline are subject to interpretation. For example, subjective variations in detection of arousals can lead to variations in scoring hypopneas related to arousals. Since arousals can vary in their intensity and subsequent autonomic responses, (Azarbarzin et al. SLEEP 2014;37(4):645-653) they are not always detected by current scoring methods. The threshold visual intensity that causes different scorers to score arousals varies considerably, with some scoring arousals with minimal, equivocal changes in EEG whereas others score arousals only when the changes are unequivocal. When arousals are generally intense this is not a problem but when arousal changes are mild, large differences in AHI can arise. While the AASM scoring rules require that only arousal lasting $3 \mathrm{~s}$ be scored, the rules do not specify the minimum time difference between an arousal following a hypopnea. This can also can lead to variations in scoring arousals and ultimately to scoring hypopneas associated with arousals.

\section{Attempts at reducing variability Identification of factors affecting scoring:}

A decade after the Chicago criteria, in an attempt to standardize definitions used by sleep laboratories and researchers, the American Academy of Sleep Medicine (AASM) published the AASM Manual for the Scoring of Sleep and Associated Events in 2007. This manual defined a hypopnea as a $30 \%$ reduction in airflow, as measured by the nasal pressure transducer flow signal, with a concomitant $4 \%$ drop in oxygen saturation; alternatively, a hypopnea was also defined as a $50 \%$ or greater decline in the flow signal associated with a 3\% drop in oxygen saturation and/or an EEG arousal lasting at least $3 \mathrm{~s}$ in duration. (Iber et al., 2007) Controversy regarding the best definition led to the adoption of both definitions in the scoring manual; the first being referred to as rule " $4 \mathrm{~A}$ " (or "recommended") (Fig. 2) and the latter as rule "4B" (or "alternative") (Fig. 3).

However, the use of the recommended vs. alternative definitions of hypopnea led to highly variable apnea/hypopnea indices. Ruehland et al. scored the same 323 consecutive sleep studies using different hypopnea definitions and found considerable variability in the median apnea-hypopnea index (AHI, $8.3 \mathrm{vs}$. 14.9 ) as well as the hypopnea index (HI, 2.2 vs. 7.2) using the recommended and alternative definitions, respectively. (Ruehland et al., 2010) Greater than half of the inconsistencies in AHI was due to the inclusion of arousals in the alternative definition, and a quarter due to the reduction of the desaturation 


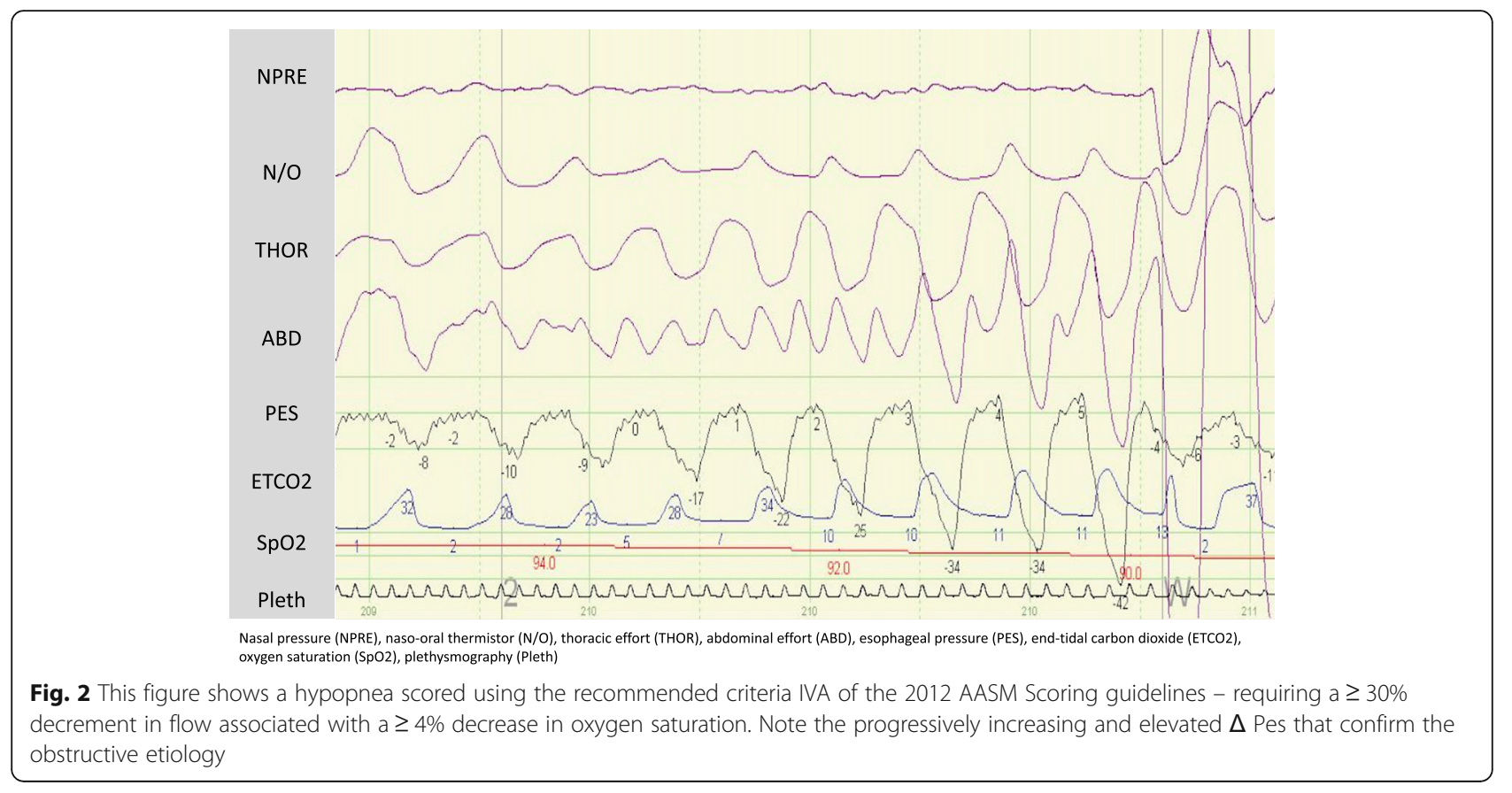

requirement from 4 to 3\%. (Ruehland et al., 2010) This translated to differences in the identification and classification of sleep apnea in the same patient. Hence, further clarification, with consideration of the clinical implications, was sought and is outlined below.

i) Effect of the arousal criterion on hypopnea scoring and classification of severity of sleep apnea The association between the arousal index and cardiovascular morbidities is not as robust as that of oxygen desaturation indices, below. However, correlations have been shown between the arousal index and hypertension (Sulit et al., 2006) as well as white matter disease in the elderly. (Ding et al., 2004) In fact, the Cleveland Family Study showed a greater correlation of hypertension risk with the arousal index than with oxygen desaturation. This may, in part, be due to the activation of the sympathetic nervous system when arousals occur during sleep, (Loredo et al., 1999; Somers et al., 1993) and the resultant sleep fragmentation leads to clinically significant symptoms. (Bonnet, 1986; Thomas, 2006; Guilleminault et al., 2009) With respect to scoring, Guilleminault et al. showed that using criteria $4 \mathrm{~A}$ to score hypopneas (i.e., a $30 \%$ flow reduction with $4 \%$ oxygen desaturation, without consideration of arousals) would have missed $40 \%$ of patients identified using the criteria incorporating arousals and who were responsive to positive airway

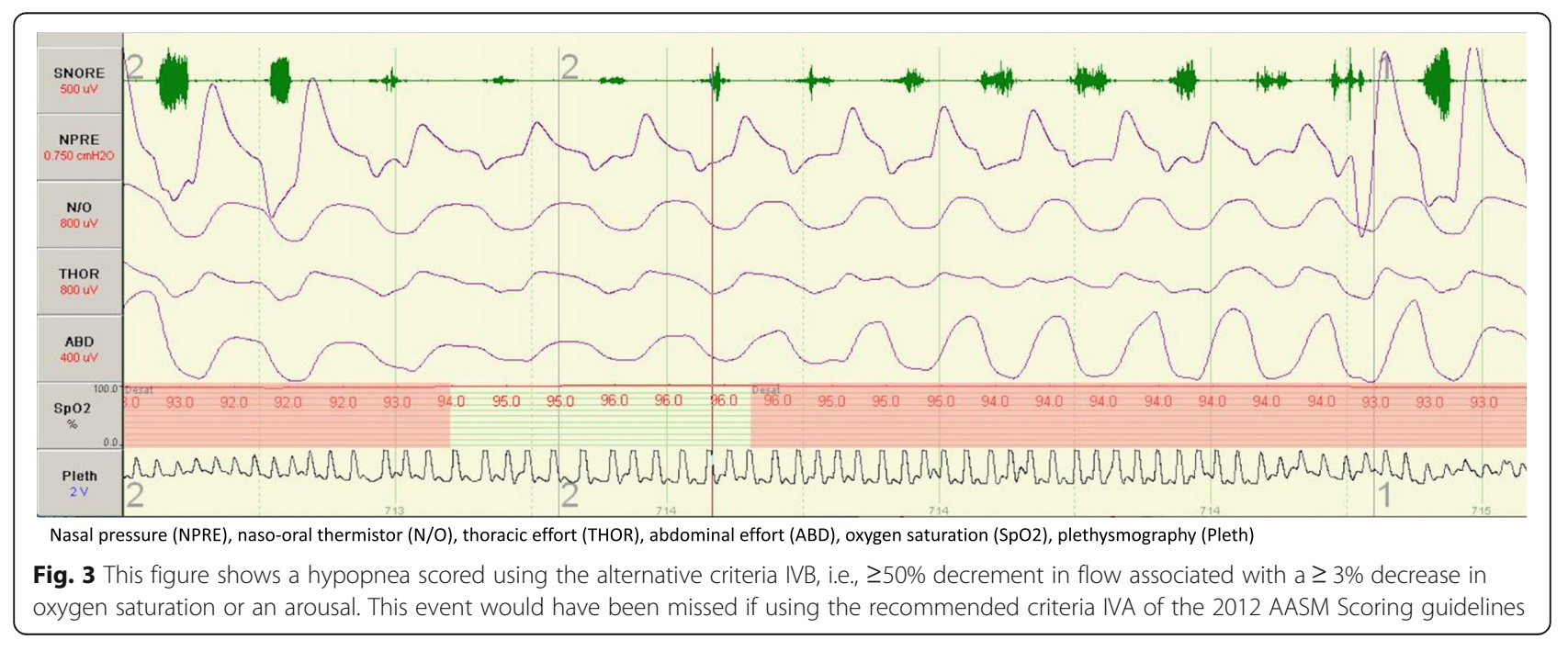


pressure (PAP) therapy (with both reductions in AHI and sleepiness). (Guilleminault et al., 2009)

ii) Effect of oxygen criterion There are clear, strong associations between obstructive respiratory events and cardiovascular events, stroke, and hyperglycemia, regardless of the $\mathrm{SpO} 2$ reduction criteria (3\% vs. $4 \%$ ) used. (Berry et al., 2012a) In addition, the correlation between AHIs scored with $3 \%$ oxygen desaturation and $4 \%$ oxygen desaturation was $>0.95$ (Redline et al., 2000), showing excellent concordance. Hence, a 3\% reduction criteria was recommended in the update to the scoring manual.

Of note, however, in 2015, Myllymaa et al. examined the effects of different oxygen desaturation threshold (ODT) levels on the AHI of 54 patients (Myllymaa et al., 2016). Hypopneas were defined as a decrement in airflow of $\geq 30 \%$ for over 10 s along with one of the following: $\quad$ an $\quad$ ODT $\geq 2 \% \quad$ (ODT2\%), ODT $\geq 3 \% \quad$ (ODT3\%), $\mathrm{ODT} \geq 4 \%$ (ODT $4 \%), \mathrm{ODT} \geq 5 \%$ (ODT5\%) or ODT $\geq 6 \%$ (ODT6\%). Not only was there a significant increase in the median AHI with ODT3\% vs. ODT4\% (6.5 events/ hr.; $p=0.003)$, different ODT's resulted in patients being classified under different categories of AHI severity. Using ODT3\% instead of ODT4\% resulted in a $44 \%$ increase (from 29.4 to $73.5 \%$ ) in the number of patients with moderate or severe OSA (AHI $\geq 15$ ). Thus, any changes in ODT, although slight, could result in significant differences in AHI, which could in turn, result in highly variable classifications of disease severity. (Myllymaa et al., 2016)

iii) Effect of flow reduction criterion Hypopneas defined with either $30 \%$ decrements in flow or $50 \%$ decrement in flow, if resulting in a desaturation or an arousal, carried clinical consequences, be it disrupted sleep, daytime sleepiness, or cardiovascular morbidity. However, a hypopnea based only on desaturation criteria alone (without arousals), would miss much clinically significant disease, as noted above.

iv) Calibration model for apnea-hypopnea indices: Impact of alternative criteria for defining hypopneas Analysis of 6441 polysomnograms showed that AHI values were sensitive and changed substantially depending on the hypopnea criteria used. (Ho et al., 2015) Also, there was greater concordance (or "stability") in AHI between the two hypopnea definitions as AHI increased above 30 , but greater variability (or "divergence") at lower AHIs. (Ho et al., 2015) Additionally, in 2 Spanish cohorts of 1116 women and 939 elderly individuals, the prevalence of an AHI $\geq 30$ events/h increased by $14 \%$ when using AHI with 3\% desaturation plus arousal criterion (AHI3\%a), compared to the AHI using 4\% (AHI4\%) desaturation criterion. (Campos-Rodriguez et al., 2016) The percentage of women with an AHI $<5$ events/h decreased from $13.9 \%$ with AHI4 to $1.1 \%$ with the AHI3\%a definition; almost one-third (31\%) of the investigated subjects moved from normal to OSA labels or vice versa. Moreover, the proportion of moderate $(15 \leq \mathrm{AHI}<30$ per hour) and severe (AHI $\geq 30$ per hour) OSA changed 13.5 and $10 \%$, respectively, depending on the hypopnea definition used. (Farre et al., 2015) Thus, although using different hypopnea criteria may not make a significant difference in OSA diagnosis for patients with more severe disease (AHI > 30), it could result in the misclassification of disease at lower AHI levels.

\section{Standardization of scoring}

These findings expounded the need for further standardization. The 2012 update to the scoring manual attempted to do just that, refining the definition of hypopnea to a $30 \%$ decrease in airflow lasting at least $10 \mathrm{~s}$ and associated $\mathrm{a} \geq 3 \% \mathrm{SpO} 2$ desaturation or an arousal. (Berry et al., 2012b) In addition, it included consensus definitions for obstructive and central hypopneas for the first time. From previous operational definitions used in heart failure with an obstructive event, obstructive hypopneas required any of the following indicators relative to baseline: paradoxical thoraco-abdominal movement, snoring, and inspiratory flattening of the flow signal whereas central hypopneas required the absence of all of these indicators (Fig. 4). Simply put, an obstructive hypopnea was a reduction in flow secondary to increased resistance of the upper airways (i.e., obstruction), whereas a central hypopnea was a result of decreased effort, not increased resistance (Fig. 5). However, the differences between central and obstructive hypopneas were not validated using esophageal catheter pressure changes, a gold standard measure of respiratory effort. Iber cautioned that given the substantial evidence supporting interaction between central and obstructive events, more emphasis should be placed on identifying causes such as heart failure, sleep disruption, and hypoxemia, rather than just distinguishing between obstructive and central events. (Iber, n.d.)

Randerath compared polysomnography (PSG) and esophageal manometry in 41 patients suspected of having sleep apnea; hypopneas were independently discriminated by blinded investigators based on either esophageal pressure or the visual PSG-based algorithm (presence or absence of flattening of the flow curve, paradoxical breathing effort, termination of the hypopnea, position of the arousal, and correlation with sleep stages). (Randerath et al., 2013) Of the 1837 scorable hypopneas, 1175 (64\%) could be further defined by esophageal pressure and 1812 (98.6\%) by the PSG-based algorithm; notably, evaluation of hypopneas using esophageal pressure was limited by 


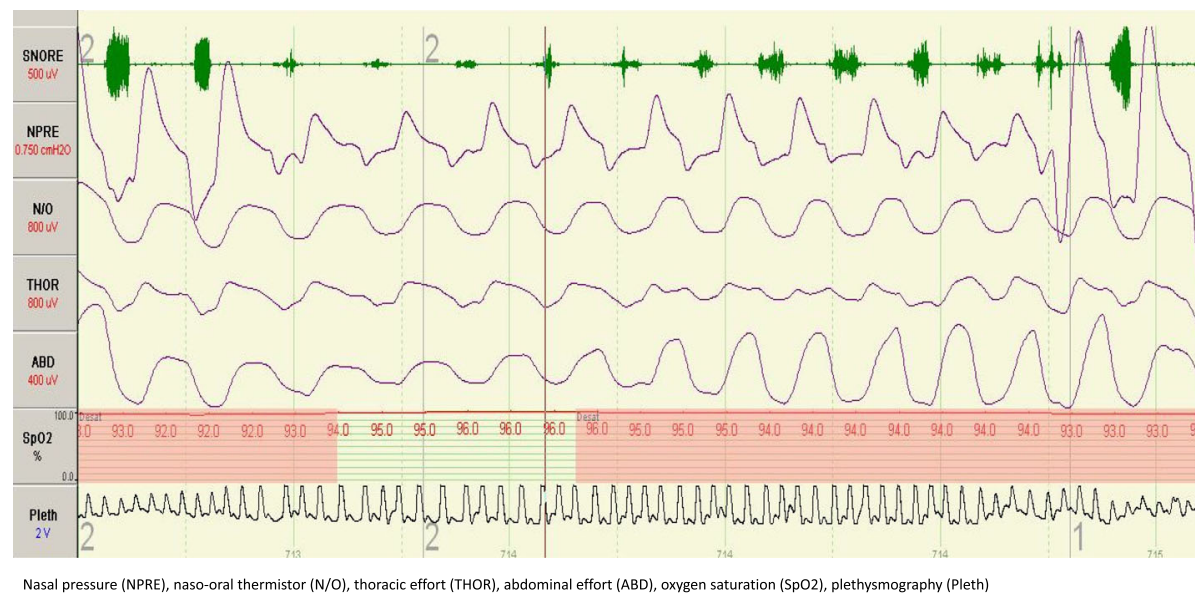

Fig. 4 An obstructive hypopnea. A hypopnea is classified as an obstructive hypopnea if the event meets all criteria for hypopnea and signs of obstruction (snoring, flow limitation, crescendo effort, or paradoxical breathing) are seen during the event

poor signal quality and artifact. Of those hypopneas that could be differentiated with both methods, using esophageal pressure as a reference, the PSG-based algorithm correctly defined $76.9 \%$ of central and $60.5 \%$ of obstructive hypopneas. However, because the esophageal manometry was not interpretable in $36 \%$ of their cases, the accuracy of a combined logic for hypopnea definition was only $68 \%$. Thus, although $77 \%$ of central hypopneas were correctly identified, nearly $40 \%$ of obstructive events were misclassified. (Randerath et al., 2013) Thus, variability in the definitions of hypopneas has led to re-classification of the type and severity of OSA.

In a retrospective study, PSGs of 112 consecutive patients for suspected OSA were re-scored for respiratory events using either 2007 AASM recommended (AASM2007Rec), 2007 AASM alternate (AASM2007Alt), Chicago criteria (AASM1999), or 2012 AASMrecommended (AASM2012) respiratory event criteria (Duce et al., 2015). The median AHI using AASM2012 definitions, was approximately 90\% greater than the AHI obtained using the AASM2007 recommended criteria, approximately $25 \%$ greater than the AASM2007Alt AHI, and approximately 15\% lower than the AASM1999 AHI. These changes increased OSA diagnoses by approximately 20 and 5\% for AASM2007Rec and AASM2007Alt, respectively. Minimal changes in OSA diagnoses were observed between AASM1999 and AASM2012 criteria. Differences between the AASM2007 using recommended criteria and AASM2012 hypopnea indices were

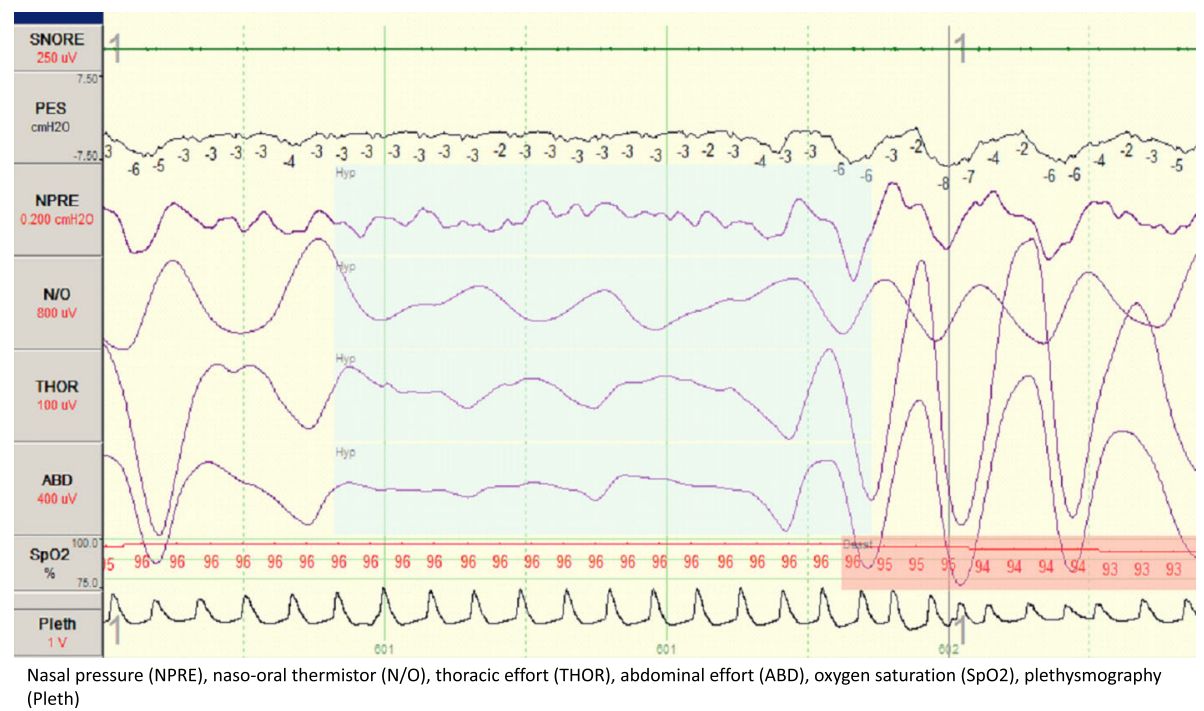

Fig. 5 A central hypopnea lacks the obstructive features seen in Fig. 4. The lack of elevated Pes values also confirms the central etiology of the hypopnea 
predominantly due to the change in desaturation levels required.

Results from such studies point to the growing importance of finding consistent methods for scoring hypopneas. Approaches designed to "calibrate AHI thresholds to the event definitions employed" or create equations to measure AHI specific to the technology in different laboratories have been considered. (Ho et al., 2015)

\section{Clinical factors determining the type of hypopnea}

Although the diagnostic value of apnea-hypopnea indices (AHIs), as determined by different hypopnea definitions, has been evaluated by investigators, it is as yet unclear what determines the type of obstructive respiratory event an individual will have. Are there physiologic characteristics that predetermine whether an individual will have primarily apneas or primarily hypopneas? What underlying differences lead to some individuals having hypopneas associated with oxygen desaturations while others have hypopneas terminating in arousals? The literature detailing this, outlined below, is sparce.

\section{Determinants of arousal-based vs. desaturation-based hypopneas}

Tsai et al. reported that regardless of the hypopnea criteria used to define sleep apnea, there were no significant differences in patient characteristics [age, sex, body mass index (BMI), and neck circumference], or in consequent Epworth Sleepiness Scale, time spent at an $\mathrm{SaO} 2$ below 90\%, arousal index, or apnea index between patients with predominantly arousal-based hypopneas versus those with desaturation-based hypopneas. (Tsai et al., 1999) No patient characteristics predicted the type of hypopnea, regardless of which hypopnea scoring method was used; however, while the addition of arousal-based scoring criteria for hypopnea caused only small changes in the AHI, OSA defined solely by an AHI value increased the prevalence of OSA. (Tsai et al., 1999)

\section{Determinants of hypopneas vs. apneas}

\section{i) Effect of BMI}

In a retrospective study of 90 adults with OSA, comparing two groups with body mass indices (BMI) $\geq 45$ vs. $\mathrm{BMI}<35$, matched for age and gender, the hypopnea-toapnea ratio (HAR) was significantly higher in the BMI $\geq 45$ group (38.8 \pm 50.7$)$ compared to the BMI $<35$ group (10.6 \pm 16.5$), p=0.0006$. (Mathew \& Castriotta, 2014) The hypopnea index, but not the apnea index, was also higher in the BMI $\geq 45$ vs. BMI $<35$ group $(28.7 \pm 28.6$ vs $12.6 \pm 8.4, p=0.0005)$, as was the AHI $(35.5 \pm 33.8$ vs $22 \pm 23, p=0.03)$. In addition, the end-tidal $\mathrm{CO} 2$ was higher in the higher BMI group. However, the hypopnea-to-apnea ratio did not appear to be influenced by the presence or absence of hypoventilation and was similar for those with or without obesity hypoventilation syndrome. (Mathew \& Castriotta, 2014) In fact, BMI was the only significant predictor of HAR (adjusted $\mathrm{r} 2=0.138 ; p=0.002)$ when adjusting for age, gender, race, and ETCO2. Of note, a small sample size may have confounded the study findings. The authors suggested that different pathophysiologic mechanisms may have been involved in the generation of apneas and hypopneas.

\section{ii) Effect of Sex Hormones}

A study of 118 patients with 'occlusive' sleep apnea syndrome, defined as daytime hypersomnolence and an AHI $>10 / h$, reported that, in women, only about $30 \%$ of respiratory events during sleep were occlusive apneas while $70 \%$ were hypopneas; conversely, in men, only $50 \%$ of events were hypopneas. The authors highlighted that both premenopausal and postmenopausal women had more hypopneas than apneas and "some of the most severely affected women were never observed to have complete cessation of airflow during sleep". (Leech et al., 1988) Notably, there were fewer sleep disordered breathing events associated with oxygen desaturation in women than men $(p<0.003) ; 19$ women did not experience oxygen desaturation at all, and only three had a total of nine episodes of apnea, whereas 20 men accounted for 264 episodes of nocturnal oxygen desaturation or abnormal breathing. (Bloch et al., 1979)

Thus, gender differences exist in the prevalence of hypopneas, and these may be conferred by differences in upper airway anatomy or control of ventilation. The latter may be attributed to hormonal differences that in turn modify ventilatory responsiveness during sleep. Rowley et al. showed that the determinants of the change in end-tidal $\mathrm{CO}_{2}$ at the apnea threshold included sex and menopausal status, with changes in end-tidal $\mathrm{CO}_{2}$ at the apnea threshold highest in premenopausal women $(4.6+/-0.6 \mathrm{mmHg})$, with no difference between the postmenopausal women $(3.1+/-0.5 \mathrm{mmHg})$ and men (3.4+/- $0.7 \mathrm{mmHg}$ ) (Rowley et al., 2006). Hormone replacement therapy increased the change in end-tidal $\mathrm{CO}_{2}\left(\mathrm{CO}_{2}\right.$ reserve) at the apnea threshold from $2.9+/-0.4 \mathrm{mmHg}$ to $4.8+/-0.4 \mathrm{mmHg}(P<.001)$ indicating that estrogens and progestins stabilize breathing in women during non-rapid eye movement sleep. (Rowley et al., 2006) Moreover, studies suggest that testosterone increases the risk for central events during sleep in men. (Zhou et al., 2003; Chowdhuri et al., 2013)

Thus, although no patient characteristics can determine the predominant type of hypopnea (arousal- vs. desaturation-based) an individual may have, obesity 
and female sex may be associated with hypopneapredominant OSA, rather than apnea-dominant.

\section{Clinical consequences of hypopneas Impact of differing definitions on clinical outcomes}

The immediate consequences of hypopneas do not appear to differ from those of apneas. In 39 sleep apnea patients who underwent polysomnography, 80 events/subject were evaluated for clinical consequences - i.e., oxygen desaturation of $\geq 4 \%$ from the baseline, EEG arousal, and an increase in heart rate by $6 \mathrm{bpm}$. (Ayappa et al., 2005) Both apneas and hypopneas were not significantly different in frequency for oxygen desaturation $(78 \%$ vs. $54 \%$, respectively) arousals (63\% vs.47\%, respectively) and associated increase in heart rate ( $73 \%$ vs. $55 \%$, respectively). In contrast, of the events with minimal (25-50\%) amplitude reduction, only $25 \%$ caused desaturation, $42 \%$ arousal, and $42 \%$ heart rate increase. No specific consequence occurred after every event. Thus, the immediate consequences of individual respiratory events (oxygen desaturation, EEG arousal and heart rate) overlapped and were not specific to any particular event. The same may not hold true for excessive daytime sleepiness or for long term cardiovascular sequelae.

i) Excessive daytime sleepiness Hosselet et al. observed that the respiratory disturbance index (RDItotal), calculated from the sum of apneas, hypopneas and flow limitation events regardless of the level of desaturation or arousal (Hosselet et al., 2001), predicted daytime sleepiness. In this study, the highest sensitivity and specificity in separating patients with excessive daytime sleepiness (EDS) from patients without EDS (non-EDS) was provided by the RDItotal. For RDItotal, the optimal combination of sensitivity and specificity was obtained at a cutoff value of 18 events/h. However, the cutoff value of 5/h for the AHI per AASM results in sensitivity of $100 \%$ but specificity for EDS of only $15 \%$.

Similarly, Ciftici et al. studied 90 patients who had an AHI $>5 / h$, scored according to the hypopnea definition of the AASM (Ciftci et al., 2004). The records of these patients were scored according to different hypopnea definitions (hypopnea-arousal, hypopnea-desaturation, hypopnea-effort). AHI (AASM), AHI (arousal), AHI (desaturation), and AHI (effort) were determined. Patients' daytime sleepiness was evaluated by the Epworth Sleepiness Scale $(>10)$. When all of three major symptoms (snoring, observed apnea, and daytime sleepiness) were found in a patient's history, the term "clinical OSAS" was applied. ESS was strongly correlated with each index. In addition, an AHI-AASM cutoff value $>5$ had the highest sensitivity and specificity from the viewpoint of separation between EDS and non-EDS, and also between clinical OSAS and nonclinical OSAS. (Ciftci et al., 2004)
Chervin \& Aldrich noted that the rate of apneas as opposed to the rate of hypopneas had a greater impact on the degree of excessive daytime sleepiness in patients with OSA (Chervin \& Aldrich, 1998). In 1146 subjects (30\% females), the mean number of apneas per hour of sleep (AI) was $14.3 \pm 27.0$ and the mean number of hypopneas per hour of sleep (HI) was $16.5 \pm 16.1$. A regression model showed that the AI explained $9.6 \%$ of the variance in mean sleep latency (MSL) $(p \leq 0.0001)$ on Mean Sleep Latency Tests, after controlling for total sleep time, but the HI explained only $5.4 \%(\mathrm{p} \leq 0.0001)$ of the variance. When AI, HI, and TST (total sleep time) were included in a single multiple-regression model, AI explained $8.3 \%$ of the variance in MSL and $\mathrm{HI}$ explained $4.0 \%$ ( $p<0.0001$ for each). The AHI during supine sleep (recorded in a subgroup of $n=169$ subjects), the rate of apneas $(n=1146)$, and the rate of obstructive apneas were useful in explaining variation in measured levels of sleepiness; however, rates of hypopneas and central apneas were not as useful. The minimum recorded oxygen saturation $(n=1097)$ was as important as the AHI to the level of sleepiness. (Chervin \& Aldrich, 1998)

ii) Metabolism In 2656 subjects from the Sleep Heart Health Study, hypopneas, even with mild degrees of oxygen desaturation of $2-3 \%$, were associated with fasting hyperglycemia, independent of multiple covariates. Hypopneas were further stratified on the degree of associated oxyhemoglobin desaturation into: $0.0-1.9 \%, 2.0-2.9 \%, 3.0-3.9 \%$, and $\geq 4.0 \%$ reductions in $\mathrm{SaO} 2$. Hypopneas based solely on the arousal criteria were not identified. The adjusted cumulative odds ratios for the hypopnea index (HI) and impaired fasting glucose were 1.15 (95\% CI: 0.90-1.47), 1.44 (95\% CI: 1.09-1.90), 2.25 (95\% CI: $1.59-3.19)$ and 1.47 (95\% CI: 1.13-1.92) respectively. (Stamatakis et al., 2008)

iii) Stroke Association between incident stroke and OSA using a hypopnea definition of $\geq 3 \%$ oxygen desaturation has been reported (Redline et al., 2010; Shahar et al., 2001) and may be somewhat stronger than the association with coronary heart disease or heart failure. This association of stroke and OSA may be mediated through ischemic pathways. Potential mechanisms: Andreas et al. simulated obstructed breaths using the Muller maneuver (generating high negative intrathoracic pressures against an obstruction) and showed a significant reduction in blood flow to the middle cerebral artery (MCA) during the period of obstruction, in conjunction with a drop in flow across the mitral and aortic valves. (Andreas et al., 1991) Using Doppler sonography, Netzer et al. showed that blood flow through the MCA was significantly reduced (i.e., > 50\% reduction in velocity) more frequently with obstructive hypopneas (76\%) and obstructive apneas (80\%) than with central apneas $(14 \%)(\mathrm{p} \leq 0.0001)$; the level of reduced 
blood flow during obstructive apneas vs. obstructive hypopneas was not significantly different. However, there was a significant association between MCA blood flow reduction and the duration of obstructive hypopnea $(p<0.05)$, which was not seen with obstructive apneas or central apneas, although mean event durations were similar $(18.1 \pm 6.5 \mathrm{~s}$ for hypopnea, $17.2 \pm 5.9 \mathrm{~s}$ for central apneas, and $14.8 \pm 5.0 \mathrm{~s}$ for obstructive apneas; $p=0.3$ ). Similarly, a statistically significant correlation $(p<0.05)$ was seen between the fall in oxygen saturation with obstructive hypopnea and reduction in MCA blood flow, not seen with central or obstructive apneas. (Netzer et al., 1998) Hence, the occurrence of MCA blood flow reduction increases as the duration of the obstructive hypopnea increases and its associated drop in oxygen saturation increases.

iv) Cardiovascular disease In a cohort of 6106 adults from the Sleep Heart Health Study hypopneas with $\geq 4 \%$ oxygen desaturations were independently associated with cardiovascular disease, whereas hypopneas with less than a $4 \%$ desaturation or arousal only were not associated with prevalent cardiovascular disease, after controlling for apnea index, age, sex, race, body mass index, waist circumference, neck circumference, total cholesterol, smoking status, and hypertension. (Punjabi et al., 2008)

Mehra et al. found significant associations between SDB and the risk of atrial fibrillation and complex ventricular ectopy (CVE) amongst 2911 elderly men without heart failure where hypopneas were defined by a desaturation criterion of $\geq 3 \%$. However, whether hypopneas predicted arrthymias was not investigated. The authors compared central vs. obstructive forms of sleep disordered breathing, and found that central sleep apnea was more strongly associated with atrial fibrillation (Odds Ratio 2.69, 95\% CI: 1.61-4.47) than CVE (OR 1.27, 95\% CI: 0.97-1.66) while OSA was associated with CVE, especially when associated with hypoxia; those in the highest hypoxia category had an increased odds of CVE (OR 1.62, 95\% CI: 1.23-2.14) compared with those with the lowest associated hypoxia. (Mehra et al., 2009)

Proposed mechanisms for the arrhythmic potential of apneas and hypopneas include intermittent hypoxia leading to increased oxidative stress, systemic inflammation, and sympathetic activity; repetitive blood pressure elevations secondary to sympathetic activation; and excessive intrathoracic pressure changes leading to mechanical stress on the heart and blood vessel walls (including large caliber vessels such as the aorta). (Camen et al., 2013; Kohler \& Stradling, 2010)

In patients with congestive heart failure (CHF), the criteria used to define hypopnea significantly influenced the AHI and the prevalence of sleep-disordered breathing (SDB). (Ward et al., 2013) The number of patients with $\mathrm{CHF}$ in whom SDB was diagnosed, using an AHI cutoff of $\geq 15 / \mathrm{h}$, increased by $16 \%$ using the AASM 'alternative' hypopnea rule ( $\geq 50 \%$ reduction in airflow with $\geq 3 \%$ oxygen desaturation or arousal) compared with the 'recommended' hypopnea scoring rule $(\geq 50 \%$ decrease in nasal airflow with $\mathrm{a} \geq 4 \%$ oxygen desaturation). Median AHI increased from 9.3/h to 13.8/h (median difference 4.6/h) and SDB prevalence increased from 29 to $46 \%$ with the AASM alternative scoring rule $(p<0.001)$. However, classification of SDB as OSA or central sleep apnea was not significantly altered by the hypopnea scoring rules.

Recent large scale studies in the non-sleep literature (McEvoy et al., 2016; Yu et al., 2017) boldly called into question the benefit of treating sleep apnea on cardiovascular outcomes and death. Although riddled with confounders such as non-adherence to PAP therapy, (McEvoy et al., 2016; Yu et al., 2017) different types of sleep apnea being treated (central vs. obstructive, (Yu et al., 2017) different modes of PAP therapy used, (Yu et al., 2017) and different diagnostic criteria for sleep apnea, (McEvoy et al., 2016) these studies raise important questions on the validity of comparing data using different recording and scoring methodologies.

Of the ten studies reviewed in Yu's meta-analysis (which included the McEvoy study), only 2 used any AASM criteria for scoring hypopneas, and though published in 2012 (Kushida et al., 2012) \& 2015 (Huang et al., 2015), both of these used the 1999 Chicago Criteria. One study from Spain (Barbe et al., 2012) used a modification of the 2012 AASM criteria (scoring hypopneas with $50 \%$ decrement in flow associated with a $4 \%$ oxygen desaturation) while another (Bradley et al., 2005) scored hypopnea as a $50 \%$ decrement in flow only (without a consequence). The remaining six studies used cardiopulmonary or respiratory polygraphy, which could not measure arousals, so any arousal-based hypopneas would have been missed. Of these limited channel studies, three used a $4 \%$ oxygen desaturation index (ODI) of $>7.5$ (Craig et al., 2012; McMillan et al., 2014) or $>12$ (4\%-drops from baseline/hour) (McEvoy et al., 2016) to diagnose sleep apnea; one (Parra et al., 2015) used a "discernible reduction in airflow or thoracic motion lasting $>10$ seconds and associated with a cyclical dip in $\mathrm{SaO} 2$ of $>3 \%$ " and calculated the AHI based on time in bed. In the remaining 2 studies (Cowie et al., 2015; Peker et al., 2016), scoring criteria were not clearly defined.

This raises many unanswered questions and reflects the current dilemmas. How did differences in diagnostic criteria affect the overall interpretation of the metaanalysis? Would the conclusions have been the same if there was a standardized definition of the disorder? Is it conceivable that treatment of apnea-predominant versus 
hypopnea predominant sleep apnea responded differently to PAP therapy? We currently do not have answers to these important questions.

v) Mortality In the clinical Spanish cohorts, AHI $\geq 30$ events/h was associated with increased cardiovascular mortality risk in women after adjusting for multiple covariates, regardless of the AHI4\%, AHI3\% or AHI3\%arousal hypopnea definition, whereas in elderly individuals the mortality risk was higher in those diagnosed using the AHI $4 \%$ and AHI3\% definitions but not using the AHI3\%a definition. (Campos-Rodriguez et al., 2016)

\section{Summary \& recommendations}

\section{A. Technical specifications}

While a number of studies have investigated the physiology and clinical significance of hypopneas, the data are sparse and inconclusive, mainly because the definitions and diagnostic methods have varied across studies. Thus, there remains a crucial gap in knowledge regarding the clinical presentation and prognosis of hypopneas. A clear, standard, and consistent definition of hypopnea is vital to this understanding. How can we claim that sleep apnea has consequences if the disorder itself is not clearly defined?

To this end, we recommend that the following specific, concrete recommendations be incorporated into the scoring guidelines:

i) Clear definition of, or guidance on, determination of baseline values for flow or $\mathrm{SpO}_{2}$. With today's technological advancements, digital methods to determine these, especially when the pre-event signals are unstable, could be helpful to avoid subjectivity.

ii) Criteria for identification of poor or unreliable signals (e.g., EEG, flow or $\mathrm{SpO} 2$ signals) and guidance on when to exclude these from the calculation of respiratory events or sleep time.

iii) Clear guidelines on arousal criteria that minimize subjectivity and bias.

iv) Specifications on the use of sensors that meet specific performance calibration criteria.

\section{Clinical impact}

Few studies have reported on the impact of the different definitions of hypopneas on chronic medical conditions. Also, studies evaluating the clinical impact of these variable definitions of respiratory events on cardiovascular or neurocognitive sequelae are lacking. Specifically, whether combinations of respiratory events, hypoxia and EEG arousals have variable physiological effects on daytime sleepiness, cardiovascular morbidity and mortality cannot be ascertained from these studies.
There are no data available regarding effects of sleep hypopneas in patients with asthma, COPD or other lung and/or neuromuscular diseases. Whether treatment of 'hypopnea-predominant' OSA leads to reduced cardiovascular morbidity or mortality or metabolic and neurocognitive dysfunction is also not known. And, although studies suggest that sleep apnea may be related to adverse clinical consequences such as cardiovascular disease, stroke, abnormal glucose metabolism, excessive daytime sleepiness, and increased mortality; further research is still needed to determine the effect that treating sleep apnea has on these condition.

\section{Conclusion}

Notwithstanding the numerous attempts at standardizing the scoring rules, the qualitative nature of scoring flow via visual inspection causes inter-observer variability, and the semi-quantitative sensors (thermistors, nasal prongs, or thoraco-abdominal bands) used to obtain uncalibrated signals for flow or effort, all lead to a level of uncertainty when scoring hypopneas. And several unanswered questions still remain regarding the final impact of using these variable hypopnea definitions for the diagnosis of OSA. Therefore, we emphasize the importance of standardizing the scoring of hypopneas across all sleep labs, regardless of their status of accreditation by the AASM.

Future research needs to focus on carefully delineating the pathophysiological significance and long-term clinical implications of the various hypopnea definitions and hypopneas per se on neurocognitive, cardiovascular and metabolic outcomes.

\section{Abbreviations \\ AASM: American Academy of Sleep Medicine; AHI: Apnea-Hypopnea Index; Al: Apnea Index; Cl: Confidence Interval; COPD: Chronic Obstructive Pulmonary Disease; CVE: Complex Ventricular Ectopy; EDS: Excessive daytime sleepiness; EEG: Electroencephalogram; EMG: Electromyogram; ETCO2: End- tidal Carbon Dioxide; HAR: Hypopnea-to-apnea Ratio; HI: Hypopnea Index; IL- 6: Interleukin-6; MCA: Middle Cerebral Artery; MSL: Mean Sleep Latency; ODT: Oxygen Desaturation Index; OSA: Obstructive Sleep Apnea; \\ OSAS: Obstructive Sleep Apnea Syndrome; PSG: Polysomnography; RDI: Respiratory Disturbance Index; SAHS: Sleep Apnea-Hypopnea Syndrome; $\mathrm{SaO} 2 / \mathrm{SpO} 2$ : Oxygen saturation; SDB: Sleep disordered breathing; \\ SE: Standard Error; TST: Total Sleep Time}

Funding

No funding was provided for the development of this manuscript.

\section{Availability of data and materials}

Not applicable. This manuscript is a review of the literature. All articles that were reviewed and referenced are available on PubMed.

\section{Author's contributions}

All authors participated in the review of the literature and in the writing of this manuscript. All authors read and approved the final manuscript.

Ethics approval and consent to participate Not applicable. 


\section{Competing interests}

The authors declare that they have no competing interests.

\section{Publisher's Note}

Springer Nature remains neutral with regard to jurisdictional claims in published maps and institutional affiliations.

\section{Author details}

'VA Ann Arbor Heathcare Center and University of Michigan, 2215 Fuller Rd, Ann Arbor, Ml 48105, USA. ${ }^{2}$ Oakland University, Rochester, MI, USA. ${ }^{3}$ John D. Dingell VA Medical Center and Wayne State University, Detroit, MI, USA.

\section{Received: 23 October 2017 Accepted: 7 May 2018}

\section{Published online: 23 May 2018}

\section{References}

Andreas S, et al. Doppler echocardiographic analysis of cardiac flow during the Mueller manoeuver. Eur J Clin Investig. 1991;21(1):72-6.

Ayappa I, et al. Immediate consequences of respiratory events in sleep disordered breathing. Sleep medicine. 2005:6(2):123-30.

Barbe $F$, et al. Effect of continuous positive airway pressure on the incidence of hypertension and cardiovascular events in nonsleepy patients with obstructive sleep apnea: a randomized controlled trial. JAMA. 2012;307(20):2161-8.

Berg S, et al. Comparison of direct and indirect measurements of respiratory aifflow: implications for hypopneas. Sleep. 1997;20(1):60.

Berry RB, et al. Rules for scoring respiratory events in sleep: update of the 2007 AASM manual for the scoring of sleep and associated events. J Clin Sleep Med. 2012a;8(5):597-619.

Berry RB, et al. The AASM manual for the scoring of sleep and associated events Rules, Terminology and Technical Specifications. Darien, Illinois: American Academy of Sleep Medicine; 2012b.

Bloch A, et al. Sleep apnea, hypopnea and oxygen desaturation in normal subjects. N Engl J Med. 1979:300:513-7.

Bonnet MH. Performance and sleepiness as a function of frequency and placement of sleep disruption. Psychophysiology. 1986;23(3):263-71.

Bradley TD, et al. Continuous positive airway pressure for central sleep apnea and heart failure. N Engl J Med. 2005:353(19):2025-33.

Iber C, Ancoli-lsrael S, Chesson AL Jr. Quan SF for the American Academy of Sleep Medicine. The AASM manual for the scoring of sleep and associated events: rules, terminology and technical specifications. 1st ed. Westchester, L: American Academy of Sleep Medicine; 2007.

Campos-Rodriguez F, et al. Impact of different hypopnea definitions on obstructive sleep apnea severity and cardiovascular mortality risk in women and elderly individuals. Sleep Med. 2016;27-28:54-8.

Chervin RD, Aldrich MS. Characteristics of apneas and hypopneas during sleep and relation to excessive daytime sleepiness. Sleep. 1998;21(8):799-806.

Chowdhuri S, et al. Testosterone conversion blockade increases breathing stability in healthy men during NREM sleep. Sleep. 2013;36(12):1793-8.

Ciftci TU, Kokturk O, Ozkan S. Apnea-hypopnea indexes calculated using different hypopnea definitions and their relation to major symptoms. Sleep and Breathing. 2004;8(03):141-6.

Cole P, Haight JS. Posture and the nasal cycle. Annals of Otology, Rhinology \& Laryngology. 1986;95(3):233-7.

Cowie MR, et al. Adaptive servo-ventilation for central sleep apnea in systolic heart failure. N Engl J Med. 2015;373(12):1095-105.

Craig $\mathrm{SE}$, et al. Continuous positive airway pressure improves sleepiness but not calculated vascular risk in patients with minimally symptomatic obstructive sleep apnoea: the MOSAIC randomised controlled trial. Thorax. 2012;67(12):1090-6.

Ding J, Nieto F, Beauchamp N Jr. Sleep-disordered breathing and white matter disease in the brainstem in older adults. Sleep. 2004;27(3):474-9.

Duce B, Milosavljevic J, Hukins C. The 2012 AASM respiratory event criteria increase the incidence of hypopneas in an adult sleep center population. $J$ Clin Sleep Med. 2015;11(12):1425-31.

Farre R, et al. A step forward for better interpreting the apnea-hypopnea index. Sleep. 2015;38(12):1839-40.

Gould G, et al. The sleep hypopnea syndrome. Am Rev Respir Dis. 1988:137(4):895-8.

Guilleminault C, Hagen C, Huynh N. Comparison of hypopnea definitions in lean patients with known obstructive sleep apnea hypopnea syndrome (OSAHS). Sleep and Breathing. 2009;13(4):341-7.

Ho V, et al. Calibration model for apnea-hypopnea indices: impact of alternative criteria for hypopneas. Sleep. 2015;38(12):1887-92.
Hosselet J-J, et al. Classification of sleep-disordered breathing. Am J Respir Crit Care Med. 2001:163(2):398-405.

Iber C. Are We Ready to Define Central Hypopneas? Sleep. 2013;36(3):305-306. https://doi.org/10.5665/sleep.2434.

Iber C. Are we ready to define central hypopneas? Sleep. 36(3):363-8.

Iber $C$, et al. The AASM manual for the scoring of sleep and associated events: rules, terminology and technical specifications: American Academy of Sleep Medicine; 2007.

Kohler M, Stradling JR. Mechanisms of vascular damage in obstructive sleep apnea. Nat Rev Cardiol. 2010;7(12):677-85.

Kushida CA, et al. Effects of continuous positive airway pressure on neurocognitive function in obstructive sleep apnea patients: the apnea positive pressure longterm efficacy study (APPLES). Sleep. 2012;35(12):1593-602.

Leech JA, et al. A comparison of men and women with occlusive sleep apnea syndrome. Chest. 1988:94(5):983-8.

Loredo JS, et al. Relationship of arousals from sleep to sympathetic nervous system activity and BP in obstructive sleep apnea. CHEST Journal. 1999;116(3):655-9.

Loube DI, et al. Indications for positive airway pressure treatment of adult obstructive sleep apnea patients: a consensus statement. Chest. 1999:115(3):863-6.

Mathew R, Castriotta RJ. High hypopnea/apnea ratio (HAR) in extreme obesity. J Clin Sleep Med. 2014;10(4):391-6.

McEvoy RD, et al. CPAP for prevention of cardiovascular events in obstructive sleep apnea. N Engl J Med. 2016;375(10):919-31.

McMillan A, et al. Continuous positive airway pressure in older people with obstructive sleep apnoea syndrome (PREDICT): a 12-month, multicentre, randomised trial. Lancet Respir Med. 2014;2(10):804-12.

Mehra R, et al. Nocturnal arrhythmias across a spectrum of obstructive and central sleep-disordered breathing in older men: outcomes of sleep disorders in older men (MrOS sleep) study. Arch Intern Med. 2009;169(12):1147-55.

Moser NJ, et al. What is hypopnea, anyway? Chest. 1994;105(2):426-8.

Myllymaa $\mathrm{K}$, et al. Effect of oxygen desaturation threshold on determination of OSA severity during weight loss. Sleep and Breathing. 2016;20(1):33-42.

Netzer $\mathrm{N}$, et al. Blood flow of the middle cerebral artery with sleep-disordered breathing correlation with obstructive hypopneas. Stroke. 1998;29(1):87-93.

Parra $\mathrm{O}$, et al. Efficacy of continuous positive airway pressure treatment on 5-year survival in patients with ischaemic stroke and obstructive sleep apnea: a randomized controlled trial. J Sleep Res. 2015;24(1):47-53.

Peker $Y$, et al. Effect of positive airway pressure on cardiovascular outcomes in coronary artery disease patients with nonsleepy obstructive sleep apnea. The RICCADSA randomized controlled trial. Am J Respir Crit Care Med. 2016;194(5):613-20

Punjabi NM, et al. Sleep-disordered breathing and cardiovascular disease: an outcomebased definition of hypopneas. Am J Respir Crit Care Med. 2008;177(10):1150-5.

Randerath WJ, et al. Evaluation of a noninvasive algorithm for differentiation of obstructive and central hypopneas. Sleep. 2013;36(3):363-8.

Redline S, Sanders M. Hypopnea, a floating metric: implications for prevalence, morbidity estimates, and case finding. Sleep. 1997;20(12):1209-17.

Redline $\mathrm{S}$, et al. Effects of varying approaches for identifying respiratory disturbances on sleep apnea assessment. Am J Respir Crit Care Med. 2000:161(2):369-74.

Redline $\mathrm{S}$, et al. Obstructive sleep apnea-hypopnea and incident stroke: the sleep heart health study. Am J Respir Crit Care Med. 2010;182(2):269-77.

Rowley J, et al. The determinants of the apnea threshold during NREM sleep in normal subjects. Sleep. 2006;29(1):95-103.

Ruehland W, et al. The New AASM Criteria for Scoring Hypopneas: Impact on the Apnea Hypopnea Index. Sleep 32: 150-157, 2009. Year Book of Pulmonary Disease. 2010;2010:244-5.

Shahar E, et al. Sleep-disordered breathing and cardiovascular disease: crosssectional results of the sleep heart health study. Am J Respir Crit Care Med. 2001:163(1):19-25.

Somers VK, et al. Sympathetic-nerve activity during sleep in normal subjects. N Engl J Med. 1993;328(5):303-7.

Stamatakis $\mathrm{K}$, et al. Fasting glycemia in sleep disordered breathing: lowering the threshold on oxyhemoglobin desaturation. Sleep. 2008;31(7):1018-24.

Sulit L, Storfer-Isser A, Kirchner H. Differences in poly-somnography predictors for hypertension and impaired glucose tolerance. Sleep. 2006:29(6):777-83.

Thomas RJ. Sleep fragmentation and arousals from sleep-time scales, associations, and implications. Clin Neurophysiol. 2006;117(4):707-11.

Tsai WH, et al. A comparison of apnea-hypopnea indices derived from different definitions of hypopnea. Am J Respir Crit Care Med. 1999;159(1):43-8. 
Ward NR, et al. The effect of respiratory scoring on the diagnosis and classification of sleep disordered breathing in chronic heart failure. Sleep. 2013;36(9):1341-8.

Whyte K, et al. Accuracy and significance of scoring hypopneas. Sleep. 1992; 15(3):257-60.

Yu J, et al. Association of Positive Airway Pressure with Cardiovascular Events and Death in adults with sleep apnea: a systematic review and meta-analysis. JAMA. 2017:318(2):156-66.

Zhou XS, et al. Effect of testosterone on the apneic threshold in women during NREM sleep. J Appl Physiol. 2003;94(1):101-7.

Ready to submit your research? Choose BMC and benefit from:

- fast, convenient online submission

- thorough peer review by experienced researchers in your field

- rapid publication on acceptance

- support for research data, including large and complex data types

- gold Open Access which fosters wider collaboration and increased citations

- maximum visibility for your research: over $100 \mathrm{M}$ website views per year 